\title{
Influence de la viscoélasticité de la paroi sur les phénomènes classiques de coup de bélier
}

\author{
par B. Bahrar \\ E.N.S.E.T de Mohammédia, Faculté des Sciences de Rabat (Maroc)
}

\section{E. Rieutord et R. Morel}

Equipe Hydraulique I.N.S.A. Lyon, Laboratoire de Mécanique des Fluides et d'Acoustique, E.C.L., UMR CNRS 5509

\section{INTRODUCTION}

L'étude classique des phénomènes de coup de bélier en conduite est habituellement menée dans l'hypothèse d'un comportement élastique linéaire de la paroi. Or l'utilisation de plus en plus fréquente de conduites en matériaux plastiques (P.V.C., polyéthylène,...) amène à examiner l'influence du comportement viscoélastique particulier de ce type de matériaux, sur les écoulements transitoires en conduite.

Dans ce domaine, les premières études semblent être celles de Tison en 1958 [1], qui avait remarqué l'influence de la déformation retardée de la conduite sur l'écoulement transitoire associé à l'ouverture d'une vanne. De manière plus approfondie, Rieutord et Blanchard [2,3] ont développé le formalisme classique concernant les écoulements instationnaires en conduites viscoélastiques. Ultérieurement, des vérifications expérimentales ont été conduites par Güney $e t$ al. [4] [5].

Dans la présente étude, nous examinons, d'abord, la théorie classique dans sa formulation élémentaire avec ou sans prise en compte de la déformation longitudinale. Une comparaison sera ici faite avec les résultats de la mesure menée par Güney [5]. Ensuite, à titre d'extension à ce type de conduite de l'étude menée récemment en conduite élastique par Bahrar et al. [6], nous examinons le comportement réel d'une telle paroi avec prise en compte non seulement de son caractère viscoélastique mais également de son inertie, de la rotation des sections ainsi que des déformations de flexion et de cisaillement.

\section{II $\square$ RELATIONS DE BASE}

\subsection{Théorie classique unidimensionnelle}

Traditionnellement, les études des écoulements transitoires en conduite sont menées dans une formulation unidimen- sionnelle, traduisant les relations de conservation de la masse et de la quantité de mouvement moyennées sur une section, soit :

$$
\begin{gathered}
\frac{\partial(\rho S)}{\partial t}+\frac{\partial(\rho S u)}{\partial x}=0 \\
\rho \frac{d u}{d t}+\frac{\partial p}{\partial x}+\rho g \frac{\partial z}{\partial x}-\frac{4 T_{f}}{D}=0
\end{gathered}
$$

avec,

$\rho$ : masse volumique du fluide, $S$ : section de la conduite, u : vitesse du fluide,

$p$ : pression, $g:$ l'accélération de la pesanteur, $z$ : cote de la conduite, $x$ : coordonnée axiale,

$t:$ temps, $D$ : diamètre intérieur de la conduite, $T_{f}:$ terme de frottement.

A ces relations de conservation, il convient d'associer celles traduisant la variation de la masse volumique $\rho$ et la déformation $\varepsilon$ de la conduite avec la pression, soit :

$$
\begin{gathered}
\frac{d \rho}{\rho}=\frac{d p}{\kappa} \\
\varepsilon=\alpha(p(x, t)-p(x, 0)) \frac{D_{m} J(0)}{2 e} \\
+\int_{0}^{t} \alpha(p(x, t-\tau)-p(x, 0)) \frac{D_{m}}{2 e} \frac{d J(\tau)}{d \tau} d \tau
\end{gathered}
$$

où,

$\kappa$ : est le module de compressibilité du fluide,

$e:$ l'épaisseur de la paroi,

$D_{m}=(D+e):$ son diamètre moyen,

$J(t):$ la fonction fluage du matériau,

$\alpha$ : paramètre caractérisant le type d'ancrage de la conduite et qui, dans le cas d'une conduite ancrée longitudinalement, s'écrit : 


$$
\alpha=1+\frac{e^{2}}{D_{m}^{2}}+v \frac{2 e}{D_{m}}-v^{2}\left(1-\frac{e}{D_{m}}\right)^{2}
$$

où $v$ est le coefficient de Poisson, supposé constant, du matériau [8].

La déformation $\varepsilon$ de la paroi peut être considérée comme la somme $\varepsilon^{e}+\varepsilon^{r}$ de deux termes, où $\varepsilon^{e}$ est la déformation élastique instantanée, dépendant de la compliance instantanée $J(0)$ de la paroi et $\varepsilon^{r}$ la déformation retardée traduisant le fluage de paroi.

La fonction fluage $J(t)$ peut être exprimée sous la forme :

$J(t)=J_{0}+\sum_{i=1}^{n} J_{i}\left(1-\exp \left(-t / \tau_{i}\right)\right)$

correspondant à un modèle de KelvinVoigt généralisé dont la représentation (fig. I) est celle de ressorts de module $E_{i}=\frac{1}{J_{i}}$ en parallèle avec des amortis-

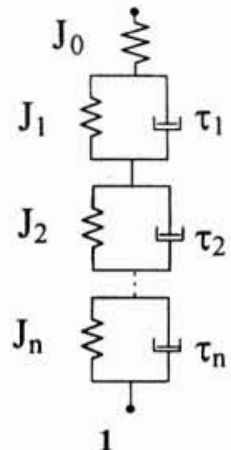
seurs de viscosité $\eta_{i}$, conduisant à une constante de temps $\tau_{i}=\frac{\eta_{i}}{E_{i}}, J_{0}$ représentant la compliance instantanée $J(0)$, inverse du module élastique instantané $E(0)$.

A noter que, si l'on pose $\varepsilon_{r}=0$, c'est-à-dire $J_{i}=0$ ou $\tau_{i}=\infty$, on retrouve le cas élémentaire classique.

\subsection{Modélisation unidimensionnelle avec prise en compte explicite de la déformation longitudinale}

On peut étendre cette formulation élémentaire pour prendre en compte, toujours en écoulement unidimensionnel, la déformation longitudinale de la conduite. On est alors conduit à un système ici linéarisé de quatre équations de conservation développé initialement, dans le cas élastique, par Otwell [9] et, appliqué ultérieurement, par Chaudhry [10] et Djemili [11].

Dans le cas viscoélastique, ce système peut s'écrire :

$$
\begin{gathered}
\rho \frac{\partial u}{\partial t}+\frac{\partial p}{\partial x}+\rho g \frac{\partial z}{\partial x}-\frac{4 T_{f}}{D}=0 \\
\frac{\partial \sigma}{\partial x}-\rho_{m} \frac{\partial v}{\partial t}-\frac{T_{f}}{e}=0 \\
\left(\frac{1}{\kappa}+\frac{\left(1-v^{2}\right) D}{e E(0)}\right) \frac{\partial p}{\partial t} \\
+\frac{\left(1-v^{2}\right) D}{e} \frac{\partial}{\partial t} \int_{0}^{t} p(t-\tau) \frac{d J(\tau)}{d \tau} d \tau-2 v \frac{\partial v}{\partial x}+\frac{\partial u}{\partial x}=0
\end{gathered}
$$

$\frac{1}{E(0)} \frac{\partial \sigma}{\partial t}-v \frac{D}{2 e E(0)} \frac{\partial p}{\partial t}+\frac{\partial}{\partial t}$

$$
+\frac{\partial}{\partial t} \int_{0}^{t}\left\{\sigma(t-\tau)-v \frac{D}{2 e} p(t-\tau)\right\} \frac{d J(\tau)}{d \tau} d \tau-\frac{\partial v}{\partial x}=0
$$

avec,

$\sigma$ : contrainte longitudinale dans le matériau,

$\rho_{m}$ : masse volumique du matériau,

$v:$ vitesse locale de déplacement axial de la conduite, $T_{f}$ : terme de frottement.

Ce système d'équations prend en compte explicitement les quatre variables $p, u, v$ et $\sigma$, en fonction de la distance $x$ et du temps $t$, incluant au travers du coefficient de Poisson $v$, le couplage dynamique entre le fluide et la paroi de la conduite.

\section{III — MÉTHODE NUMÉRIQUE DE RÉSOLUTION}

Les relations (1), (2) et (3) ou (6), (7), (8) et (9) forment un système hyperbolique pouvant être résolu par la méthode habituelle des caractéristiques $[12,13]$.

\subsection{Système classique}

On a ici trois directions caractéristiques et les relations de comptabilité correspondantes s'écrivent :

- le long des courbes caractéristiques de pentes, $\frac{\mathrm{d} x}{\mathrm{~d} t}=u \pm a$ :

$\frac{d p}{d t} \pm \rho a \frac{d u}{d t}+2 \frac{\rho a^{2}}{u \pm a}\left(u \frac{d \varepsilon^{r}}{d t} \pm a \frac{\partial \varepsilon^{r}}{\partial t}\right) \pm \rho g a \frac{\partial z}{\partial x} \mp a \frac{4 T_{f}}{D}=0$

- le long de la caractéristique $\frac{\mathrm{d} x}{\mathrm{~d} t}=0$ :

$$
\frac{\partial \varepsilon^{r}}{\partial t}=\int_{0}^{t} \frac{\alpha D_{m}}{2 e} \frac{\partial p(x, t-\tau)}{\partial t} \frac{d J(\tau)}{d \tau} d \tau
$$

où :

$$
a=\left(\rho\left(\frac{1}{\kappa}+\alpha \frac{D_{m} J(0)}{e}\right)\right)^{-1 / 2}
$$

\subsection{Modélisation avec prise en compte de la déforma- tion longitudinale}

On a ici cinq directions caractéristiques et les relations de comptabilité associées s'écrivent :

- le long des courbes caractéristiques relatives au fluide $\frac{\mathrm{d} x}{\mathrm{~d} t}= \pm C_{f}$ :

$$
\begin{aligned}
& \frac{d p}{d t} \pm \rho C_{f} \frac{d u}{d t} \pm \rho_{\mathrm{m}} C_{f} G_{f} \frac{d v}{d t}-G_{f} \frac{d \sigma}{d t}+H_{f} \frac{\partial \varepsilon_{1}^{r}}{\partial t} \\
& -E(0) G_{f} \frac{\partial \varepsilon_{2}^{r}}{\partial t} \mp C_{f}\left(\frac{4}{D}+\frac{G_{f}}{e}\right) T_{f} \pm C_{f} \rho g \frac{\partial z}{\partial x}=0
\end{aligned}
$$

- le long des courbes caractéristiques correspondant au matériau $\frac{\mathrm{d} x}{\mathrm{~d} t}= \pm C_{m}$ :

$$
\begin{aligned}
& \frac{d p}{d t} \pm \rho C_{m} \frac{d u}{d t} \pm \rho_{\mathrm{m}} C_{m} G_{m} \frac{d v}{d t}-G_{m} \frac{d \sigma}{d t}+H_{m} \frac{\partial \varepsilon_{1}^{r}}{\partial t} \\
& -E(0) G_{m} \frac{\partial \varepsilon_{2}^{r}}{\partial t} \pm C_{m}\left(\frac{4}{D}+\frac{G_{m}}{e}\right) T_{f} \pm \mathrm{C}_{\mathrm{m}} \rho g \frac{\partial z}{\partial x}=0
\end{aligned}
$$

- le long de la caractéristique $\frac{\mathrm{d} x}{\mathrm{~d} t}=0$ :

$$
\begin{gathered}
\frac{\partial \varepsilon_{r}^{1}}{\partial t}=\int_{0}^{t} v \frac{D_{m}}{2 e} \frac{\partial p(x, t-\tau)}{\partial t} \frac{d J(\tau)}{d \tau} d \tau \\
\frac{\partial \varepsilon_{r}^{2}}{\partial t}=\int_{0}^{t} \frac{\partial \sigma(t-\tau)}{\partial t} \frac{d J(\tau)}{d \tau} d \tau
\end{gathered}
$$

Dans ces relations

$C_{f}=\frac{a_{f}}{\tilde{J}} q_{1}$ correspond à la vitesse de propagation des perturbations dans le fluide, $C_{m}=\frac{a_{f}}{\tilde{J}} q_{2}$ correspond à la vitesse de propagation des déformations longitudinales de la paroi, 


$$
\begin{gathered}
G_{f}=\frac{2 v \rho C_{f}^{2}}{\rho_{m}\left(a_{m}^{2}-C_{f}^{2}\right)}, \quad G_{m}=\frac{2 v \rho C_{m}^{2}}{\rho_{m}\left(a_{m}^{2}-C_{m}^{2}\right)}, \\
H_{f}=\frac{2\left(1+v^{2}\right)}{v} \rho C_{f}^{2}+E(0) G_{f}, \quad H_{m}=\frac{2\left(1-v^{2}\right)}{v} \rho C_{m}^{2}+E(0) G_{m},
\end{gathered}
$$

où :

$$
\begin{gathered}
a_{f}=(\kappa / \rho)^{1 / 2}, a_{m}=\left(E(0) / \rho_{m}\right)^{1 / 2}, \\
\tilde{J}=\left(1+\left(1-v^{2}\right) D \kappa / e E(0)\right)^{1 / 2} \\
q_{1}=\left(\frac{1+q}{2}+\sqrt{\left(\frac{1-q}{2}\right)^{2}+\frac{v^{2} D \rho}{e \rho_{m}}}\right)^{1 / 2} \\
q_{2}=\left(\frac{1+q}{2}-\sqrt{\left(\frac{1-q}{2}\right)^{2}+\frac{v^{2} D \rho}{e \rho_{m}}}\right)^{1 / 2}
\end{gathered}
$$

et $\quad q=\left(1+\frac{D \kappa}{e E(0)}\right) \frac{a_{m}^{2}}{a_{f}^{2}}$

La résolution de ces équations de comptabilité le long des courbes caractéristiques permet de calculer numériquement en chaque point de la conduite, les valeurs de $p, u, v$ et $\sigma$ au cours du temps.

\section{C COMPARAISON DES RÉSULTATS THÉORIQUES ET EXPÉRIMENTAUX}

Pour la plupart des matériaux plastiques utilisés en hydraulique, (polyéthylène, P.V.C,...), le comportement viscoélastique est d'autant plus marqué que la température est élevée, comme le montre la figure 2 ci-contre correspondant à une conduite en polyéthylène testée au Laboratoire de Mécanique des Fluides de l'I.N.S.A de Lyon [5].

A basse température, la faible évolution de $J(t)$ explique que l'on puisse y admettre un comportement sensiblement élastique. En revanche, lorsque la température s'élève, l'effet de la viscoélasticité devient prépondérant et doit être pris en compte dans les calculs.

A titre d'exemple, nous avons comparé, sur la figure 3, le résultat de calculs faits dans l'hypothèse d'un matériau élastique et viscoélastique avec un enregistrement de coup de bélier résultant de la fermeture quasi instantanée ( $10 \mathrm{~ms})$, d'une vanne à l'aval d'une conduite en polyéthylène, ali-

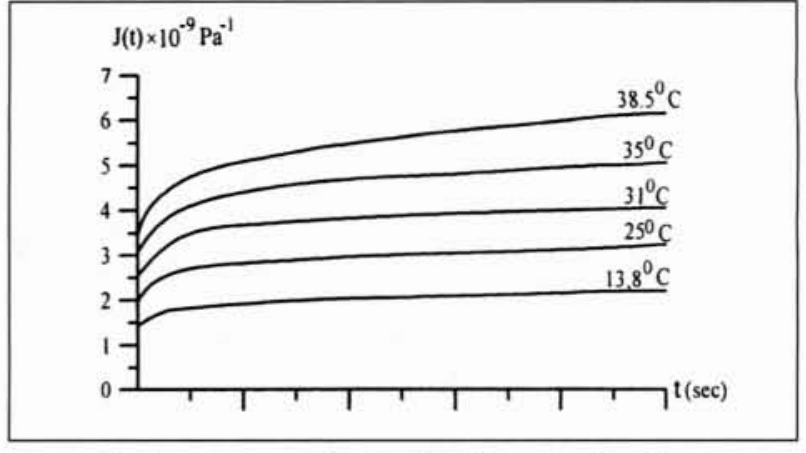

\section{Comportement viscoélastique en fonction de la température.}

mentée par un réservoir à pression constante. La conduite de type industriel a une longueur de $43,1 \mathrm{~m}$, un diamètre intérieur de $41,6 \mathrm{~cm}$ et une épaisseur de paroi de $4,2 \mathrm{~mm}$. La masse volumique $\rho_{m}$ du polyéthylène est ici de $930 \mathrm{~kg} / \mathrm{m}^{3}$.

La modélisation sous forme d'éléments de Kelvin-Voigt, de la fonction de fluage $J(t)$ de la figure 2 correspond, à la température de $25^{\circ} \mathrm{C}$ aux valeurs suivantes des différents paramètres :

$J_{0}=1,542 \times 10^{-9} \mathrm{~Pa}^{-1}, J_{1}=0,754 \times 10^{-9} \mathrm{~Pa}^{-1}$,

$J_{2}=1,046 \times 10^{-9} \mathrm{~Pa}^{-1}$,

$J_{3}=1,237 \times 10^{-9} \mathrm{~Pa}^{-1}, \tau_{1}=0,89 \times 10^{-4} \mathrm{sec}, \tau_{2}=0,0222 \mathrm{sec}$, $\tau_{3}=1,864 \mathrm{sec}$.

Les résultats présentés ci-dessus, représentent la variation de la pression à la vanne en fonction du temps. Ils correspondent respectivement à :

Graphe (1): résultats de mesure,

Graphe (2): calcul théorique en conduite élastique, dans les conditions citées ci-dessus, les pertes de charge étant celles correspondant à un régime permanent,

Graphe (3): calcul théorique en conduite viscoélastique, les pertes de charge étant également celles correspondant à un régime permanent,

Graphe (4): calcul théorique en conduite viscoélastique, toujours dans les mêmes conditions, mais avec prise en compte au niveau des pertes de charge, du caractère non permanent de l'écoulement [7]. Cette correction améliore légèrement la concordance entre théorie et expérience.

On constate que la viscoélasticité a un effet d'amortissement important, dont le calcul classique en conduite supposée élastique, ne rend pas compte.

Lorsque la température augmente, l'effet viscoélastique amortissement et étalement des perturbations — devient de plus en plus marqué comme le montrent les graphes des figures 4 et 5 , où les calculs théoriques correspondent, dans la modélisation de Kelvin-Voigt, aux valeurs suivantes des paramètres.

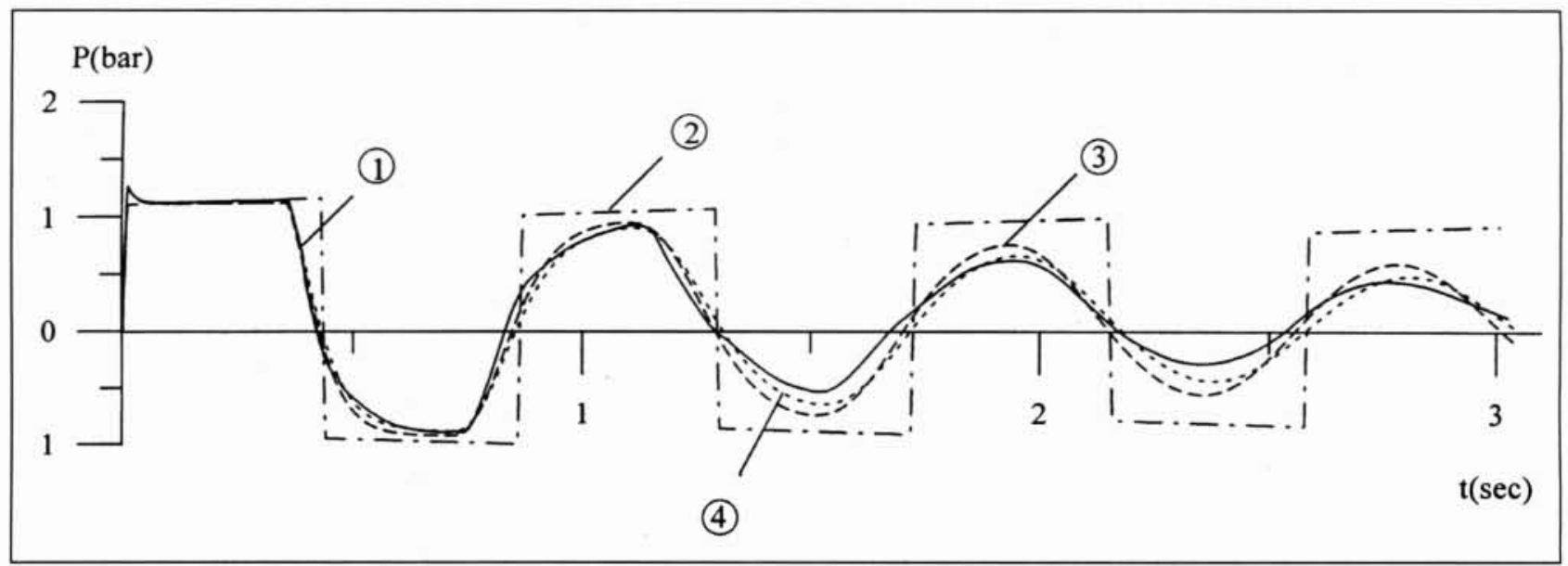

3. Comparaison théorie et expérience - température $25^{\circ} \mathrm{C}$. 


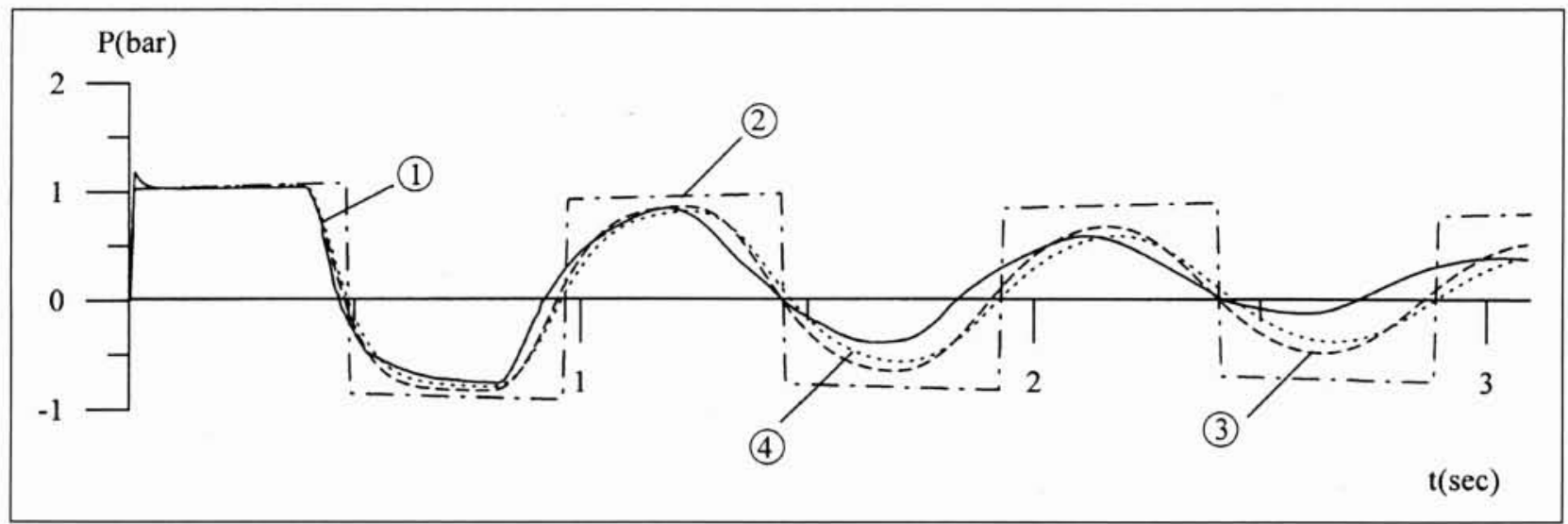

4. Comparaison théorie et expérience - température $31^{\circ} \mathrm{C}$.

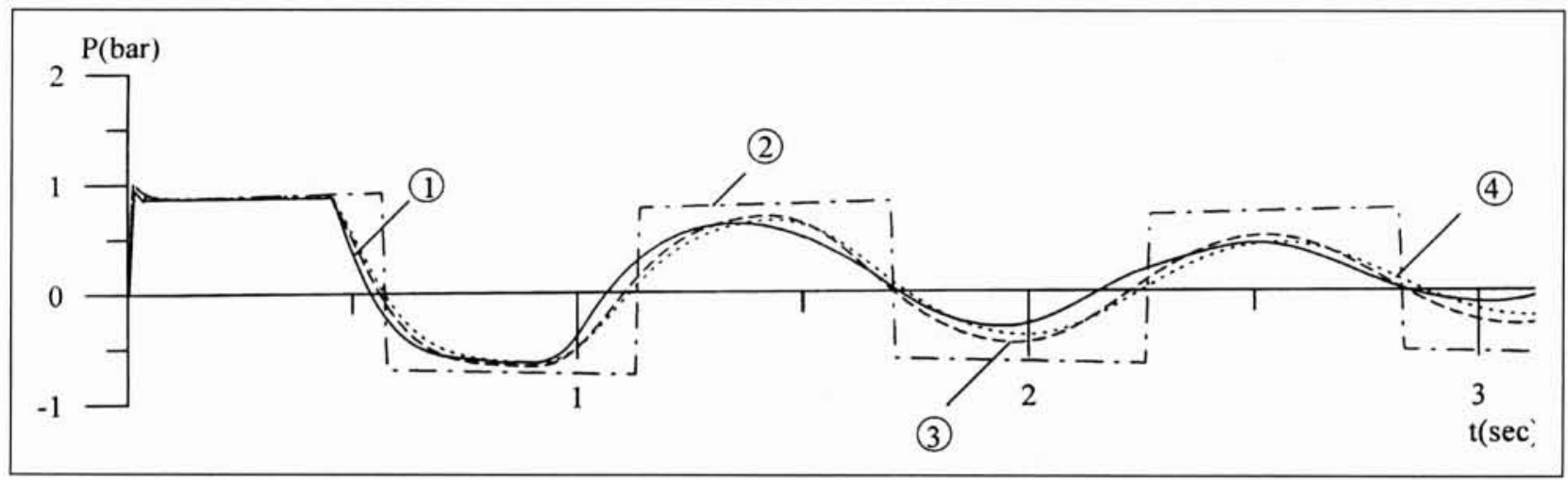

5. Comparaison théorie et expérience - température $38,5^{\circ} \mathrm{C}$.

à $31^{\circ} \mathrm{C}$ :

$J_{0}=1,791 \times 10^{-9} \mathrm{~Pa}^{-1}, J_{1}=1,009 \times 10^{-9} \mathrm{~Pa}^{-1}$,

$J_{2}=1,397 \times 10^{-9} \mathrm{~Pa}^{-1}, J_{3}=1,628 \times 10^{-9} \mathrm{~Pa}^{-1}$,

$\tau_{1}=1,15 \times 10^{-4} \mathrm{sec}, \tau_{2}=0,0221 \mathrm{sec}, \tau_{3}=1,822 \mathrm{sec}$

à $38,5^{\circ} \mathrm{C}$ :

$J_{0}=2,239 \times 10^{-9} \mathrm{~Pa}^{-1}, J_{1}=1,479 \times 10^{-9} \mathrm{~Pa}^{-1}$,

$J_{2}=2,097 \times 10^{-9} \mathrm{~Pa}^{-1}, J_{3}=3,570 \times 10^{-9} \mathrm{~Pa}^{-1}$,

$\tau_{1}=1,24 \times 10^{-4} \mathrm{sec}, \tau_{2}=0,0347 \mathrm{sec}, \tau_{3}=3,077 \mathrm{sec}$.

On constate ici également, un excellent accord théorie-expérience lorsqu'est prise en compte la viscoélasticité de la conduite.

\section{V — FORMULATION GÉNÉRALE}

Comme indiqué en introduction, nous avons examiné dans un récent article [6], une formulation générale permettant d'analyser la structure réelle des phénomènes de coup de bélier, lorsqu'on prend en compte, la déformation réelle de la conduite.

Les équations développées dans le cas élastique, s'étendent immédiatement au cas viscoélastique si l'on remarque que dans l'espace des transformées de Laplace-Carson, les lois de comportement élastique et viscoélastique liant contraintes et déformations ont la même forme [14].

En élastique, $E^{*}(s)$ est une constante simplement égale au module de Young $E$, alors qu'en viscoélastique, $E^{*}(s)=\frac{1}{J^{*}(s)}$ est la transformée de la fonction de relaxation
$E(t)$, ou de l'inverse de la transformée de la fonction de fluage $J(t)$.

Donc, si l'on applique les développements mathématiques développés en [6] en y remplaçant simplement $E^{*}(s)$, par sa valeur, on peut analyser très directement l'influence de la viscoélasticité.

A titre d'exemple, nous donnons sur les figures 6 et 7 ciaprès, en variables adimensionnalisées comme indiqué en [6], une comparaison au temps $t=20$, de la réponse à un échelon unité de pression appliqué au temps $t=0$ à l'entrée de la conduite, dans le cas élastique et viscoélastique (polyéthylène à $25^{\circ} \mathrm{C}$ ).

L'influence de la viscoélasticité de la paroi se traduit d'une part, par un amortissement des oscillations de pression et d'autre part, par des vitesses de propagation légèrement inférieures.

Dans le cas présent où le temps $t=20$, correspondant à un temps réel de $1,36 \mathrm{~ms}$ nettement supérieur au plus petit des temps de relaxation $\tau_{1}=0,089 \mathrm{~ms}$, tout se passe comme si les vitesses $C_{1}, C_{2}, a_{2}, a_{3}$ et $a_{4}$ étaient celles d'une conduite élastique de module $1 /\left(J_{0}+J_{1}\right)$, comme on peut le voir sur la figure 7 suivante où le calcul en «élastique " a été mené avec cette valeur du module.

A ce propos, on peut noter une réduction continue de la vitesse de propagation au fur et à mesure que la valeur de $t$ devient supérieure à chacun des temps de relaxation $\tau_{1}, \tau_{2}$ et $\tau_{3}$, pour finalement correspondre, comme on le verra, à celle d'un matériau élastique de module $E(\infty)=1 /\left(J_{0}+J_{2}+J_{3}\right)$.

Quant au front d'onde primaire dans le fluide de célérité $a_{1}$, son amplitude et sa vitesse de propagation, liées essentiellement à la compressibilité du fluide et à l'élasticité instantanée de la conduite, ne sont évidemment pas affectées par la viscoélasticité de la paroi comme on le verra ci-après. 

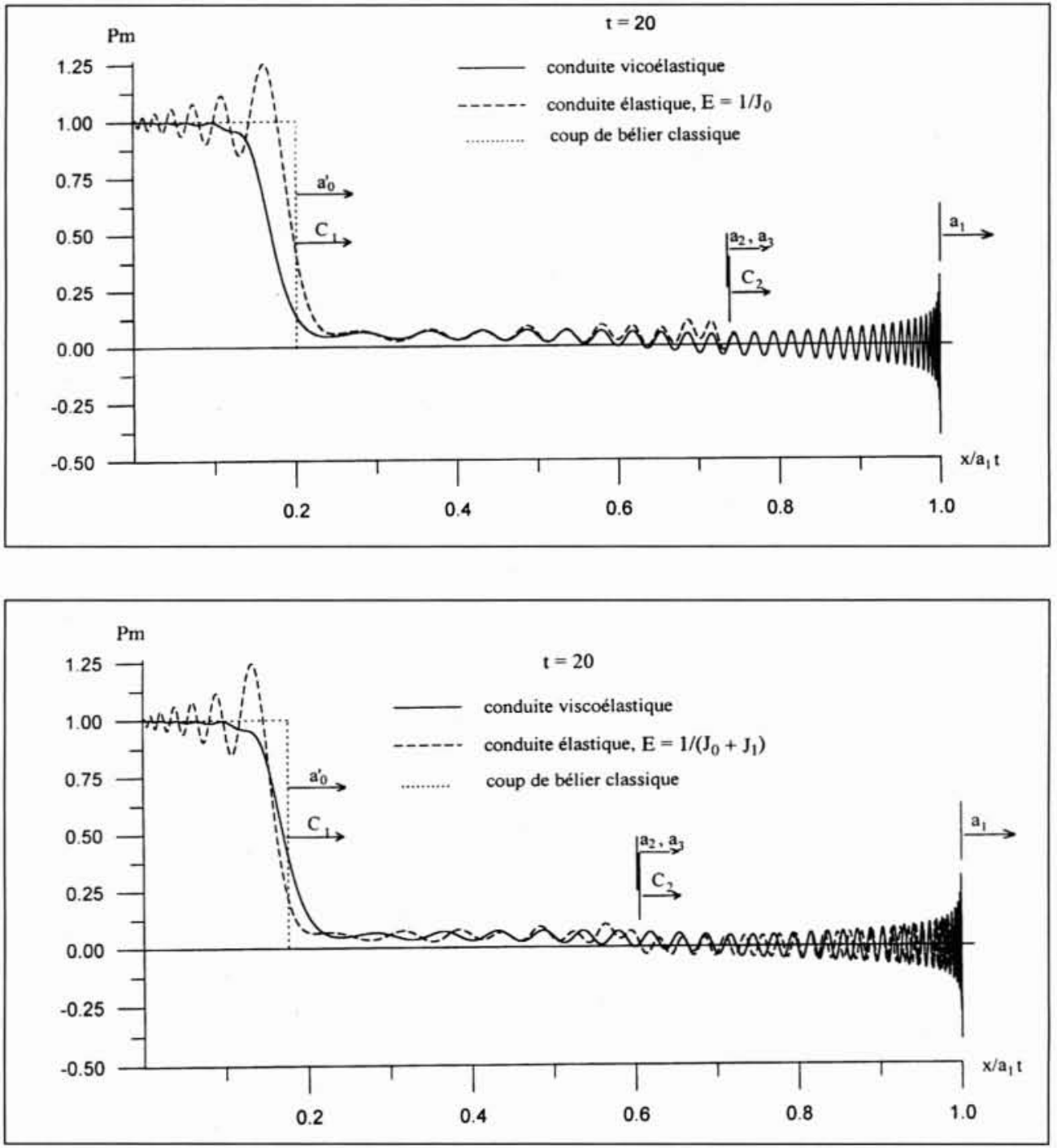

6. Répartition de la pression le long de la conduite aux temps courts (calcul en élastique avec $\left.E=1 / J_{0}\right)$.

7. Répartition de la pression le long de la conduite aux temps courts (calcul en élastique $\operatorname{avec} E=1 /\left(J_{0}+J_{1}\right)$.

\section{Analyse asymptotique}

Tout comme dans le cas élastique, on peut faire l'analyse du comportement asymptotique au voisinage des différents fronts d'onde de célérité $a_{n}$.

Pour le front d'onde primaire dans le fluide de célérité $a_{1}$, les développements analytiques au voisinage immédiat de ce front d'onde sont identiques à ceux du cas élastique, c'est-àdire :

- Dans le cas de fluide parfait :

$$
p_{m 1} \approx j_{0}\left(\sqrt{2 x a_{1}\left(t-x / a_{1}\right) / \eta}\right) H\left(t-x / a_{1}\right)
$$

où $H$ est la fonction d'Heaviside et $j_{0}$ la fonction de Bessel d'ordre 0 .

- Dans le cas de fluide visqueux :

$$
\begin{gathered}
p_{m 1}(x, t) \approx \exp \left(-x d_{2} / a_{1} r_{i}^{2} R e\right) \int_{0}^{\tau=t-x / a_{1}} \frac{x d_{1}}{2 \sqrt{\pi\left(\tau-t^{\prime}\right)^{3}}} \\
j_{0}\left(\sqrt{2 a_{1} d_{3} x t^{\prime}}\right) \exp \left(-x^{2} d_{1}^{2} / 4\left(\tau-t^{\prime}\right)\right) d t^{\prime} H(\tau)
\end{gathered}
$$

où les différents coefficients sont donnés en [6].

Il en résulte comme le montre la figure 8 , que la viscoélasticité de la conduite n'a pas d'effet sur l'amortissement du front d'onde primaire dans le fluide, l'amortissement étant à ce niveau uniquement lié à la viscosité du fluide (voir graphe à $t=100$ ).

En ce qui concerne les autres fronts d'onde qui se propagent dans le matériau de la conduite avec les célérités $a_{2}, a_{3}$ et $a_{4}$, des développements analytiques analogues conduisent aux expressions suivantes, faisant apparaître au voisinage de ces fronts d'onde, par rapport au cas élastique, un amortissement lié à la viscoélasticité de la paroi au travers de $J^{\prime}(0)$ valeur à $t=0$ de la fonction mémoire $d J(t) / d t$.

$$
p_{m_{n}} \approx B_{n} \frac{t-x / a_{n}}{x a_{n} b_{n}} \exp \left(-\gamma_{n} x\right) j_{2}\left(\sqrt{2 x a_{n} b_{n}\left(t-x / a_{n}\right)}\right) H\left(t-x / a_{n}\right)
$$

pour $\mathrm{n}=2,3,4$

où $j_{2}$ est la fonction de Bessel d'ordre 2 et $\gamma_{n}=\rho_{m}\left(1-v^{2}\right) \bar{a}_{n} \bar{a}_{0} \bar{J}^{\prime}(0)$.

La figure 9 ci-après illustre, pour la déformation de flexion, l'amortissement important introduit par la viscoélasticité de la paroi.

Comportement aux grandes valeurs de $t$ - front de diffusion Lorsque $t$ augmente et devient supérieur au plus grand des temps de relaxation, ici $\tau_{3}=1,864 \mathrm{sec}$, il ne reste plus du fait de la viscoélasticité de la paroi et éventuellement de la viscosité du fluide, que deux perturbations ayant une amplitude notable correspondant aux deux fronts de diffusion de célérités $C_{1}$ et $C_{2}$ (fig. 10). 


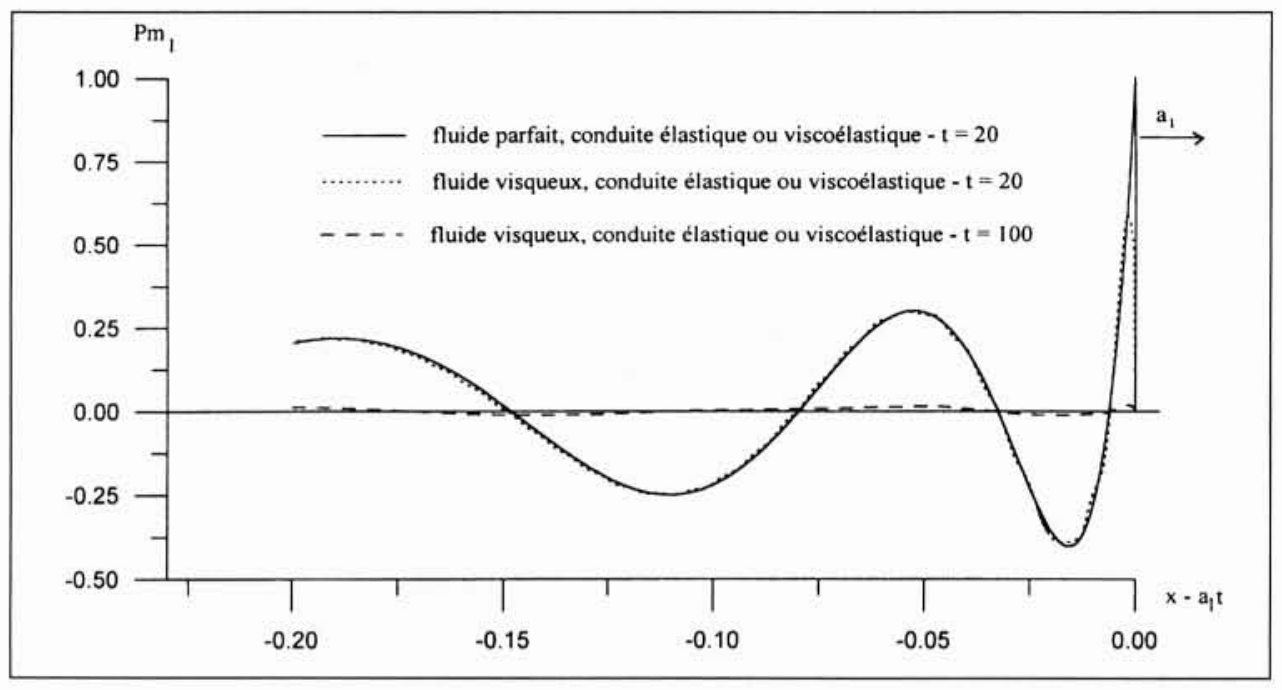

8. Front d'onde primaire dans le fluide.

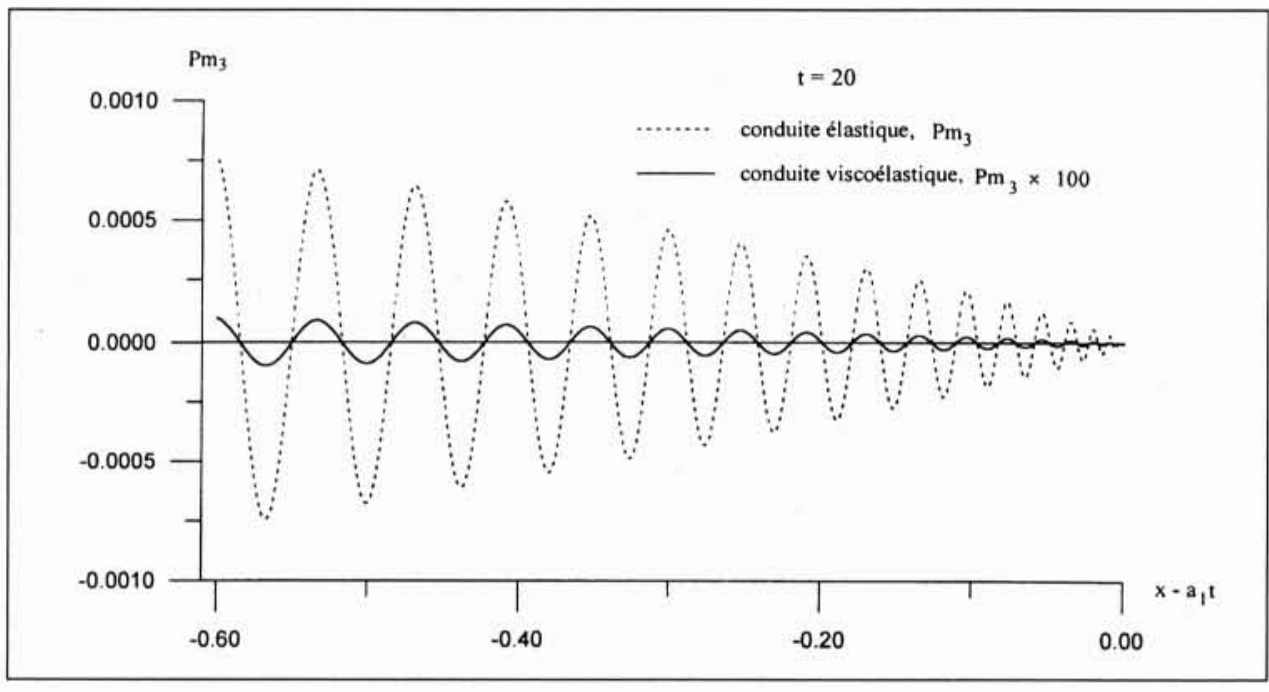

9. Front d'onde de flexion de célérité $a_{3}$.

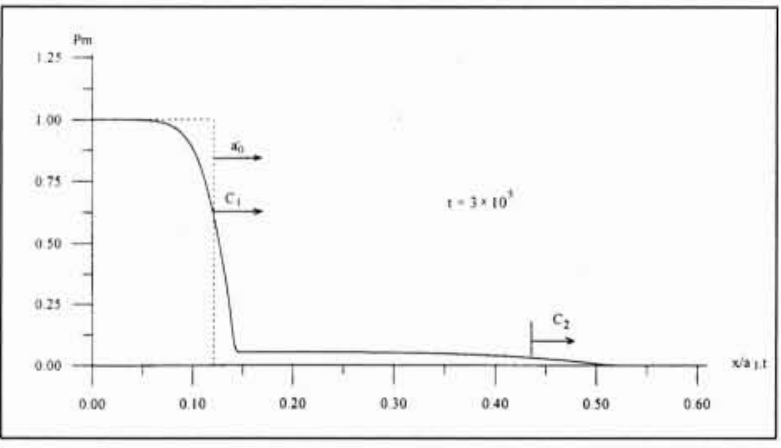

10. Répartition de la pression le long de la conduite aux temps longs.

Ces fronts de diffusion correspondent à ceux du cas élastique, mais où le module d'élasticité de la paroi a été remplacé par le module retardé $E(\infty)=\frac{1}{J(\infty)}$ où, dans la modélisation de Kelvin-Voigt, $J(\infty)=J_{0}+\Sigma J_{i}$, ce qui conduit, en variables adimensionnelles, aux célérités $C_{1}$ et $C_{2}$ telles que :

$$
C_{1}=\left(\frac{1+q}{2}+\sqrt{\left(\frac{1-q}{2}\right)^{2}+\frac{v^{2} D \rho_{f}}{e \rho_{m}}}\right)^{1 / 2},
$$

$$
C_{2}=\left(\frac{1+q}{2}-\sqrt{\left(\frac{1-q}{2}\right)^{2}+\frac{v^{2} D \rho_{f}}{e \rho_{m}}}\right)^{1 / 2},
$$

où :

$$
q=\left(1+\frac{D K}{e E(\infty)}\right) \frac{\rho_{f}}{\rho_{m}} \frac{E(\infty)}{K}
$$

a) Front de diffusion $C_{1}$

Comme en élastique, le front de diffusion se propageant à la vitesse $C_{1}$, a sensiblement même célérité que celle $a_{0}^{\prime}$ fournie par la théorie élémentaire classique où l'on prendrait comme module d'élasticité de la conduite $E(\infty)$. Quant à son amplitude, en fluide parfait, elle est de la forme :

$$
p_{m 1} \approx \frac{L_{1}}{2}\left\{1+\operatorname{erf} \frac{C_{1} t-x}{2 \sqrt{C_{1} x}} \sqrt{-\left.\frac{1}{J(\infty)} \frac{d J *(s)}{d s}\right|_{s=0}}\right\}
$$

b) Front de diffusion $C_{2}$

Le front $C_{2}$ a un comportement analogue, son évolution est donnée par:

$$
p_{m 2} \approx \frac{L_{2}}{2}\left\{1+\operatorname{erf} \frac{C_{2} t-x}{2 \sqrt{C_{2} x}} \sqrt{-\left.\frac{1}{J(\infty)} \frac{d J^{*}(s)}{d s}\right|_{s=0}}\right\}
$$




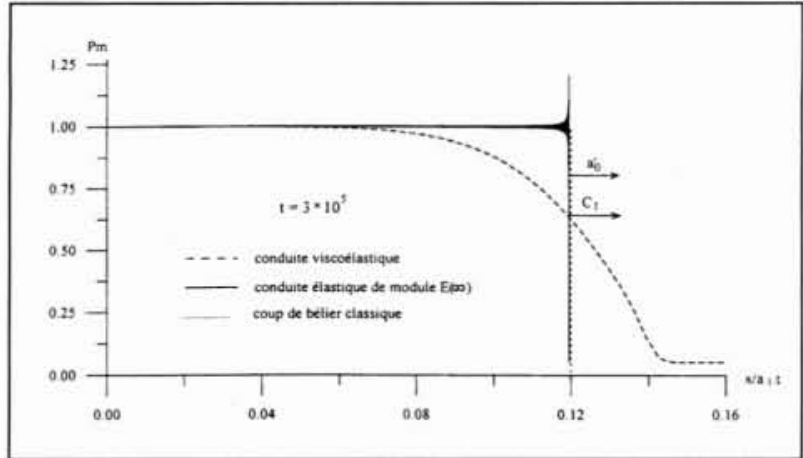

11. Evolution du front de diffusion $C_{1}\left(25^{\circ} \mathrm{C}\right)$.

Dans les expressions ci-dessus, les coefficients $L_{1}$ et $L_{2}$ sont ceux relatifs au cas élastique, mais calculés avec le module retardé $E(\infty)$, leur somme correspondant à l'échelon de pression unité appliqué à l'origine de la conduite.

Quant à la fonction erreur dont la valeur reste comprise entre -1 et +1 elle traduit, lorsque $t$ augmente, l'étalement de la perturbation initiale, étalement directement lié, au travers du terme en $\left.\frac{1}{J(\infty)} \frac{d J^{*}(s)}{d s}\right|_{s=0}$ au fluage de la paroi résultant de la viscoélasticité du matériau.

Les résultats donnés ci-dessus en fluide parfait, se retrouvent en fluide visqueux où les effets de la viscosité du fluide s'ajoutent à la viscoélasticité du matériau.

\section{Conclusion}

La présente étude montre l'influence de la viscoélasticité de paroi dans les phénomènes classiques de coup de bélier, concernant les conduites en matériau plastique tel que le polyéthylène.

La modélisation unidimensionnelle rend bien compte de l'amortissement observé expérimentalement, lié au fluage du matériau, et donne des résultats en excellent accord avec les mesures.

Si maintenant l'on prend en compte, au travers de ses différentes composantes (flexion, cisaillement,....), la déformation réelle de la paroi, on constate que la viscoélasticité introduit, outre l'amortissement précité, un étalement des fronts d'onde et fronts de diffusion associés à chacune des composantes de la déformation.

On observe également une réduction des vitesses de propagation en liaison directe avec l'évolution du module de relaxation $\mathrm{E}(\mathrm{t})$ ou de la fonction fluage $\mathrm{J}(\mathrm{t})$, caractérisant le comportement viscoélastique du matériau.

\section{RÉFÉRENCES BIBLIOGRAPHIQUES}

[1] Tison G. (1958). — Le mouvement non permanent succédant à l'ouverture d'une vanne sur une conduite en polyéthylène. Becetel, communication $\mathrm{N}^{\bullet} 3$, Université de Gand.

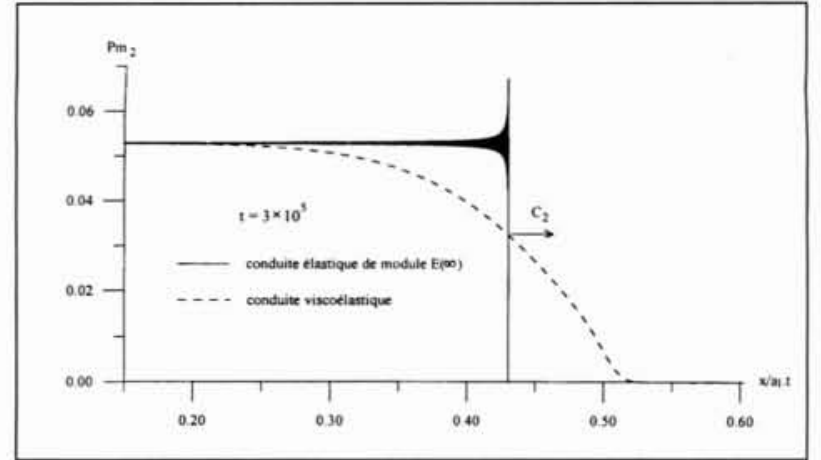

12. Evolution du front de diffusion $C_{2}\left(25^{\circ} \mathrm{C}\right)$.

[2] Rieutord E. et Blanchard A. (1972). - Influence d'un comportement viscoélastique de la conduite dans le phénomène du coup de bélier. C. R. Acad. Sc. Paris, tome 274

[3] Rieutord E. and Blanchard A. (1979). - Ecoulement non permanent en conduite viscoélastique, coup de bélier. Journal of Hydraulic research, Vol. 17, $\mathrm{N}^{\bullet} 3$.

[4] Gally M., Góney M. and Rieutord E. (1979). - An Investigation of Pressure Transients in Viscoelastic Pipes. ASME Journal of Fluids Engineering, Vol. 101.

[5] GÜNEY M. (1977). - Contribution à l'étude du phénomène de coup de bélier en conduite viscoélastique. Thèse présentée à l'Université de Lyon.

[6] Bahrar B., Rieutord E., Morel R., ZegGwagh G. (1996). - Modélisation du phénomène de coup de bélier avec prise en compte du comportement réel de la conduite. La Houille Blanche, nं 1-1998 pp. 18-25.

[7] ZielKe W. (1968). - Frequency Dependent Friction in Transient Pipe Flow. ASME, Journal of Basic Engineering, Vol. $90, \mathrm{~N}^{*} 1$.

[8] Wylie E.B., Streeter V.L. (1978). - Fluid Transients. New York, Mac Graw-Hill.

[9] OTwell R. S., - The Effect of Elbow Restraint on Pressure Transient, Ph. D. dissertation, Michigan State University, 1984.

[10] Chaudhry M.H., Walter S., Elansary A.S. (1994), Numerical and Experimental Investigation of Transient Pipe Flow. Journal of Hydraulic Research, Vol. 32, $\mathrm{N}^{*} 5$.

[11] DJemill A. (1988). - Influence de l'ancrage de la conduite, paroi élastique ou viscoélastique, sur les écoulements en régime transitoire. Doctorat $3^{\mathrm{e}}$ cycle, I.N.S.A. de Lyon.

[12] Courant A.R. et Hilbert D. (1962). - Method of Mathematical Physics, Vol. 1 et 2, Interscience Publishers, N.Y.

[13] Goldberg D.E, and WyLIE E.B. (1983). - Caracteristics Method Using Time-Line Interpolation. ASCE Journal of Hydraulic Division, Vol. 109, N* 5.

[14] Mandel J. (1966). - Cours de mécanique des milieux continus, Gauthier-Villars, Paris, Vol. 2, Annexe XXI. 